\title{
Evaluating the Effects of Intratympanic Dexamethasone and Lidocaine in Refaractory Tinnitus
}

\author{
Original \\ Article \\ Mohammad Amin Al-Morsy, Ahmed Nabil Elsamanody, Marawan Ahmed Ibrahim \\ Department of Otorhinolaryngology, Al-Azhar University Hospitals, Al-Azhar University, \\ Cairo, Egypt.
}

\begin{abstract}
Objective: To evaluate the effect of intratympanic dexamethasone and lidocaine in minimizing refractory tinnitus. Background: Tinnitus is one of the most annoying otologic problems that may affect the quality of life. Tinnitus has many varieties in its intensity and duration. The management of tinnitus includes a lot of options that could be medical therapy, psychotherapy and physiotherapy. Steroids are known to be one of lines of its treatment with variable effects, so that lidocaine could have additive effect on the treatment of idiopathic tinnitus.

Patients and Methods: This clinical trial study includes 56 people who had been diagnosed with refractory tinnitus that was diagnosed in these patients depending on failure of gingko biloba over 4 weeks. These patients were randomly divided into two groups, group A of 32 cases treated with intratympanic injection of lidocaine and dexamethasone and group B of 24 cases treated with intratympanic injection of dexamethasone. Improvement was evaluated in both groups before and after the procedure.

Results: There was statistical significant difference among studied groups comparing Tinnitus Handicap Inventory scoring before and after the intra-tympanic injection. There was non-statistical significant difference between both groups with tinnitus of low-frequency. But there was statistical significant difference between both groups with tinnitus of highfrequency.

Conclusion: Intratympanic injection of lidocaine with dexamethasone in refractory tinnitus showed significant better results especially at low frequency tinnitus. This technique was evaluated with few temporary side effects.
\end{abstract}

Key Words: Intratympanic injection, lidocaine, tinintus.

Received: 11 September 2020, Accepted: 20 December 2020

Corresponding Author: Mohammad Amin Al-Morsy, MD, Department of Otorhinolaryngology, Al-Azhar University Hospitals, Al-Azhar University, Cairo, Egypt, Tel.: 01004488602, E-mail: mohamed_amin2022@yahoo.com

ISSN: 2090-0740, 2021 Vol.22

\section{INTRODUCTION}

The definition of tinnitus is the perception of involuntary sounds either in one or both ears. It could be due to central or peripheral causes with different theoretical mechanisms. Cochlear lesion is one of the most common causes of tinnitus that could be accompanied with hearing affection $^{[1]}$.

Tinnitus may affect the quality of life causing insomnia and depression. Types of tinnitus include either objective or subjective tinnitus. Subjective tinnitus is the most common forms of annoying tinnitus that usually persists for longer period ${ }^{[2]}$.

It is hard to diagnose and treat a case of idiopathic tinnitus without any positive history data either medication history or history of cochlear trauma or noise exposure. Pure tone audiometry may help in diagnosis of any sensorineural hearing loss indicating affection of cochlea or cochlear nerve $\mathrm{e}^{[3]}$.
Many techniques were subjected to help in reduction of the tinnitus frequency or by masking the tinnitus. These techniques were developed over-years for better results. Electronic hearing aids with tinnitus masker aim to decrease the tinnitus frequency by masking the tinnitus sound. However, the success rate of these methods remains relatively variable. Either general or intratympanic medications have a major role in minimizing the annoying effect of tinnitus and decrease its effect on patient's life $\mathrm{e}^{[4]}$.

Steroids or lidocaine are main drug options used for managing idiopathic tinnitus. Many studies showed the effects of intratympanic steroid injection rather than its systemic usage because of the general side effects of steroid and the direct access of intratympanic route with higher doses reaching the cochlea ${ }^{[5]}$.

Lidocaine also can be used as another powerful option in managing tinnitus, although its damage effects on cochlear and vestibular functions. Vertigo, nausea and 
vomiting are common accompanying symptoms with the use of intratympanic lidocaine, but these symptoms may persist for short period. This aim of this study was to evaluate the intratympanic injection of lidocaine in addition with dexamethasone for patients with idiopathic subjective tinnitus ${ }^{[6]}$.

\section{PATIENTS AND METHODS:}

This prospective study was conducted on 56 patients presented refractory tinnitus at ENT Department of Al-Azhar University hospitals (AL-Hussein and Bab El-Shaaria) in the period from December 2018 to May 2020. Informed consent was taken from all patients after approval of the ethical committee of Al-Azhar University. Refractory tinnitus was diagnosed in these patients depending on failure of gingko biloba over 4 weeks to change patient's satisfaction using the tinnitus questionnaire before and after drug medication.

Diagnosis of patients included in our study depending on patients complaining of tinnitus in spite of 4 weeks of treatment and exclusion of any systemic or topical objective causes that are usually presented with tinnitus as following steps:

- Initially patients were assessed by full history taking and otoscopic examination

- Audiologic testing as pure tone audiometry for hearing evaluation, tympanometry and tinnitogram, auditary brainstem response

- Magnetic resonance imaging were done to exclude any retrochochlear lesions

- $\quad$ Systemic causes of tinnitus were also excluded as hypertension or disturbed thyroid functions.

Tinnitus was evaluated depending on Tinnitus Handicap Inventory (THI) (25 questions with score 0 for No, score 2 for sometimes and score 4 for Yes) that had 5 grades of tinnitus severity (Grade I for slight handicap, Grade II for mild handicap, Grade II for moderate handicap, Grade IV for severe handicap and Grade V for catastrophic handicap). This score was evaluated at the first visit and 4 weeks after the last injection visit ${ }^{[7]}$. Patient satisfaction was measured using visual analogue score after the injection to differ patients who were satisfied or fairly satisfied or unsatisfied.

Patients were assigned into two lists of cases, the first list of 32 cases were managed using intratympanic dexamethasone (Dexamethasone disodium phosphate, $5 \mathrm{mg} / \mathrm{ml}$ ) and lidocaine (40 $\mathrm{mg}$ in $1 \mathrm{ml}$ saline) as group $\mathrm{A}$ and the second list of 24 cases were managed using intratympanic injection of dexamethazone only as group B. But only $1.2 \mathrm{ml}$ of the prepared material was injected intratympanic.
Under local anathesia using lidocaine spray (10\%), examination of the tympanic membrane was done. Anterosuperior quadrant of the tympanic membrane was punctured by spinal anesthesia needle and intratympanic injection was done with the prepared material. After injection, the patient was instructed not to swallow or move his head for 30 minutes. This technique was done twice with 2 week in between. Any additional manifestations or side effects were recorded in follow-up visits.

\section{Statistical Analysis}

Descriptive statistics included the mean value and standard deviation plus minimum and highest frequency. The t-test was used for quantitative analysis. The Chisquare test was used for the analysis of qualitative data. The SPSS 22.0 program was used for statistical analysis. So, the p-value was considered significant as the following: Probability ( $P$-value): P-value 0.05 was considered insignificant.

\section{RESULTS:}

A total of 56 patients were considered in our study, majority of these patients $(73.2 \%)$ were female. The mean age of patients was $41.6 \pm 5.2$ years. The mean duration of refractory tinnitus was $28.3 \pm 9.2$ months.

The mean threshold of hearing for group $\mathrm{A}$ and B were $22.4 \pm 6.3 \mathrm{~dB}$ and $25.2 \pm 8.6 \mathrm{~dB}$ respectively. (Table 1) shows the frequency of tinnitus either high or low frequency in each group.

Table 1: Patient's hearing and tinnitus assessment

\begin{tabular}{|c|c|c|c|}
\hline \multicolumn{2}{|l|}{ Groups } & Group A $(\mathrm{N}=32)$ & Group B $(\mathrm{N}=24)$ \\
\hline \multicolumn{2}{|c|}{ Hearing threshold } & $\begin{array}{c}\text { Mean } \pm \text { SD }= \\
22.4 \pm 6.3\end{array}$ & $\begin{array}{c}\text { Mean } \pm \text { SD }= \\
25.2 \pm 8.6\end{array}$ \\
\hline \multirow[t]{2}{*}{ Tinnitus freq. } & Low & $14(43.75 \%)$ & $10(41.7 \%)$ \\
\hline & High & $18(56.25 \%)$ & $14(58.3 \%)$ \\
\hline
\end{tabular}

There was statistical significant difference among studied groups comparing THI scoring before and after the intra-tympanic injection. (Table 2).

Table 2: THI assessment using (Student t-test)

\begin{tabular}{lcccc}
\hline THI & & $\begin{array}{c}\text { Before } \\
\text { Procedure } \\
\text { Mean } \\
\pm \mathrm{SD}\end{array}$ & $\begin{array}{c}\text { After } \\
\text { Procedure } \\
\text { Mean } \\
\pm \mathrm{SD}\end{array}$ & $p$-value \\
\hline Group A & Low & $64 \pm 6.4$ & $45.7 \pm 9.9$ & $0.0001 \mathrm{HS}$ \\
& High & $68.8 \pm 7.3$ & $54.3 \pm 10.4$ & $0.0001 \mathrm{HS}$ \\
Group B & Low & $62.6 \pm 7.7$ & $41.8 \pm 4.5$ & $0.0001 \mathrm{HS}$ \\
& High & $71.4 \pm 9.7$ & $62.4 \pm 12.6$ & $0.044 \mathrm{~S}$ \\
\hline
\end{tabular}


Table 3 shows the patient satisfaction according to visual analogue score. There was non-statistical significant difference between both groups with tinnitus of lowfrequency. But there was statistical significant difference between both groups with tinnitus of high-frequency.

Table 3: Patients' satisfaction by visual analogue score using (Chi-square test)

\begin{tabular}{lcccc}
\hline $\begin{array}{l}\text { Patient } \\
\text { satisfaction }\end{array}$ & \multicolumn{2}{c}{ Low Frequency } & \multicolumn{2}{c}{ High Frequency } \\
Group A & Group B & Group A & Group B \\
\hline Satisfied & 10 & 6 & 14 & 6 \\
$\begin{array}{l}\text { Fairly } \\
\text { satisfied }\end{array}$ & 2 & 3 & 3 & 2 \\
$\begin{array}{l}\text { Unsatisfied } \\
\quad 2\end{array}$ & 2 & 1 & 1 & 6 \\
$\quad$-value & \multicolumn{2}{c}{$0.905 \mathrm{NS}$} & \multicolumn{2}{c}{$0.038 \mathrm{~S}$} \\
\hline
\end{tabular}

There were side effects recorded after injection as dizziness, temporary stitching earache and recurrent tinnitus. Any dizziness or temporary earache was relieved after short period of follow-up (maximal after 3 days) as shown in (Table 4).

Table 4: Complications after injection technique

\begin{tabular}{lcc}
\hline Complications & Group A & Group B \\
\hline Dizziness & 15 & 3 \\
Temporary earache & 2 & 5 \\
Not relieved tinnitus & 2 & 1 \\
\hline
\end{tabular}

\section{DISCUSSION}

Tinnitus is the perception of noise in the ears which may be soft or loud without external acoustic stimulation and it may also sound like a buzzing, clicking, hiss, or roaring. This complaint may be annoying for a long period of time that interfere the quality of life. These patients are seeking for effective therapy either medical or non-medical line of management. ${ }^{[8]}$

El-Hennawi et al. studied 46 patients with idiopathic subjective unilateral tinnitus. These patients were randomly divided into two groups one was injected with lidocaine and another one was injected with methyl prednisolone. Group with intratympanic injection by lidocaine showed a lower percentage of improvement compared with methyl prednisolone group. Lidocaine was previously attempted in the management of tinnitus by the $1970 \mathrm{~s}$, and up to $82.8 \%$ improvement was reported. ${ }^{[9,10]}$

In a study conducted by She and colleagues, the improvement in subjective tinnitus either after intra-tympanic injection with prednisolone or dexamethasone were 48.6 and $37.5 \%$, respectively. However, this study defined improvement as a decrease in loudness of at least $5 \mathrm{~dB}$ after comparing tinnitus intensities obtained pretreatment and post-treatment or when persistent tinnitus became intermittent. ${ }^{[11]}$

Our study detected that there is significant patient satisfaction after 4 weeks between both groups at high frequency tinnitus while non-significant difference at low frequency tinnitus. 54 patients were included in another study and were treated with intra-tympanic dexamethasone injections. After 6 weeks, patients showed either experienced complete resolution, significant improvement or no change of tinnitus as following $34 \%, 40 \%$ and $26 \%$ respectively. At the 1-year follow-up, only two of the 54 patients studied continued to report complete resolution of symptoms. ${ }^{[12]}$

In another report, in which only nine of 52 patients completed a 5-week course of IT-LD, all nine patients showed improvement. Another study reported a similar improvement rate of $81 \%$ upon treatment of cochlear tinnitus with IT-LD in 369 patients $^{[13,14]}$.

Intra-tympanic injection is usual method used in management of otologic problems which is a safe and simple technique. Our study showed side effects recorded after injection as dizziness, temporary stitching earache and/or hearing loss. Minimal complaints which were mild and resolved spontaneously as light vertigo and otalgia were detected in a study presented by Araújo. ${ }^{[15]}$

In another study, 21 of the 23 participants who received LD reported dizziness that lasted up to $6 \mathrm{~h}$. The findings that the effects of LD injection-associated vertigo were transient warrant its possible use in treating tinnitus. Another study reported no side effects such as hearing loss, eardrum perforation, or dizziness in any patients. ${ }^{[9,11]}$

However, in another study, $20 \%$ of the patients of steroid-injected group complained of the aggravation of tinnitus. ${ }^{[16]}$

\section{CONCLUSION}

Intratympanic injection of lidocaine with dexamethasone in refractory tinnitus showed significant better results especially at low frequency tinnitus. This technique was evaluated with few temporary side effects.

\section{CONFLICT OF INTEREST}

There are no conflicts of interest. 


\section{REFERENCES}

1. Baguley D, McFerran D, Hall D. "Tinnitus". The Lancet. 382 (9904): 1600-07.

2. Shim HJ, Jun Song S, Choi AY, Hyung Lee R, Won Yoon S. Comparison of various treatment modalities for acute tinnitus. Laryngoscope 2011; 121:26192625 .

3. Wu V, Cooke B, Eitutis S, Simpson MTW, Beyea JA. Approach to tinnitus management. Can Fam Physician. 2018; 64(7):491-495.

4. Sandlin RE, Olsson RJ. Evaluation and selection of maskers and other devices used in the treatment of tinnitus and hyperacusis. Trends Amplif. 1999;4(1):626.

5. Bear ZW, Mikulec AA. Intratympanic steroid therapy for treatment of idiopathic sudden sensorineural hearing loss. Mo Med. 2014;111(4):352-356.

6. Langguth B, Salvi R, Elgoyhen AB. Emerging pharmacotherapy of tinnitus. Expert Opin Emerg Drugs. 2009;14(4):687-702.

7. 14Newman CW, Jacobson GP, Spitzer JB. Development of the Tinnitus Handicap Inventory. Arch Otolaryngol Head Neck Surg 1996;122;143-148.

8. Levine RA, Oron Y. "Tinnitus". The Human Auditory System - Fundamental Organization and Clinical Disorders. Handbook of Clinical Neurology. 2015; 129:409-31

9. El Hennawi DM, Ahmed MR, Abdelkafy W, Geneid A, Ibrahim IH. Intratympanic methylprednisolone acetate versus intratympanic lidocaine in the treatment of idiopathic subjective unilateral tinnitus of less than 1-year duration: a randomized, double-blind, clinical trial. Egypt J Otolaryngol 2017;33:564-8

10. Sakata E, Umeda Y. Treatment of tinnitus by transtympanic infusion. Auris Nasus Larynx 1976; $3: 133-138$

11. She W, Dai Y, Du X, Chen F, Ding X, Cui X. Treatment of subjective tinnitus: a comparative clinical study of intratympanic steroid injection vs. oral carbamazepine. Med Sci Monit 2009; 15:PI35-PI39.

12. Cesarani A, Capobianco S, Soi D, Giuliano DA, Alpini D. Intratympanic dexamethasone treatment for control of subjective idiopathic tinnitus: our clinical experience. Int Tinnitus J 2002; 8:111-114.

13. Sakata H, Kojima Y, Koyama S, Furuya N, Sakata E. Treatment of cochlear tinnitus with transtympanic infusion of $4 \%$ lidocaine into the tympanic cavity. Int Tinnitus J 2001; 7:46-50.

14. Podoshin L, Fradis M, David YB. Treatment of tinnitus by intratympanic instillation of lignocaine (lidocaine) 2 per cent through ventilation tubes. J Laryngol Otol 1992; 106:603-606.

15. Araujo MF, Oliveira CA, Bahmad FM Jr. Intratympanic dexamethasone injections as a treatment for severe, disabling tinnitus. Does It Work? Arch Otolaryngol Head Neck Surg 2005; 31: 113- 117.

16. Choi SJ, Lee JB, Lim HJ, In SM, Bae KH, Choung YH. Intratympanic dexamethasone injection for refractory tinnitus: Prospective placebo $\square$ controlled study. Laryngoscope. 2013; 123:2817-2822. 